Filozofska fakulteta, Univerza v Ljubljani

marija.javorbriski@ff.uni-lj.si

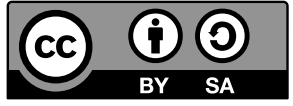

\title{
LITERATUR UND MENSCHLICHES WISSEN. ANALYSEN ZU EINER GRENZÜBERSCHREITENDEN BEZIEHUNG
}

Helena Ulbrechtová, Frank Thomas Grub, Edgar Platen, Siegfried Ulbrecht (Hgg.) (2018). Literatur und menschliches Wissen. Analysen zu einer grenzüberschreitenden Beziehung. Berlin: Kulturverlag Kadmos. ISBN 978-3-86599-360-1, 411 Seiten, $29.80 €$.

Die von Helena Ulbrechtová, Frank Thomas Grub, Edgar Platen und Siegfried Ulbrecht herausgegebene Monographie Literatur und Wissen. Analysen zu einer grenzüberschreitenden Beziehung ist eine produktive Weiterführung der noch immer aktuellen Forschungsdebatte über das Verhältnis von Literatur und Wissen, deren Lücken aufgrund der Komplexität des Themas auch das jüngst erschienene interdisziplinäre Handbuch nicht schließen konnte. Das vorliegende Buch ist das Resultat des Prager und Göteborger Projektteams, das sich über einen längeren Zeitraum mit dem Thema aus transdisziplinärer Perspektive intensiv auseinandergesetzt hat.

Das einleitende Kapitel bietet einen detaillierten Überblick über die Forschungsgeschichte zum Thema Literatur und menschliches Wissen im Spannungsfeld von Kulturund Naturwissenschaften unter besonderer Berücksichtigung der Fiktion als einer Form des Wissens. Näher vorgestellt werden $u$. a. die Forschungen des Literatur- und Kulturzentrums in Berlin, die Verhandlung des Themas in tschechischen Wissenschaftskreisen und das gemeinsame Projekt der in der vorliegenden Monographie mitwirkenden Autoren.

Sehr aufschlussreich sind Edgar Platens theoretische Ausführungen zum Verhältnis von Literatur und Wissen. Die seit der Antike forcierte Trennung des erkennenden Subjekts von der Welt führt den Überlegungen des Autors zufolge im Namen einer dem Objektivitätsideal huldigenden Wissenschaftlichkeit zu einer Reduktion der Welterkenntnis. In einer luziden Darstellung richtet er sein Hauptaugenmerk auf die Bestimmung spezifisch literarischer Wissensformen und deren Beitrag zum Wissen des Anderen.

Nach theoretischen Vorüberlegungen zum Verhältnis von Wissen und Literatur fokussiert sich Frank Thomas Grub in seinen Ausführungen auf Reisetexte aus der DDR. Anhand einer gut fundierten Analyse exemplarischer Beispiele zeigt er, wie das für 
totalitäre Staaten übliche Gutachterverfahren vor der Drucklegung von Werken das Wissen systematisch mit Blick auf die herrschende Ideologie transformiert.

Achim Küpper befasst sich mit dem bislang eher wenig erforschten Verhältnis von Literatur und Alchemie, deren Wissens- und Erkenntnisbestände als Folge aufklärerischer Bestrebungen in wissenschaftlichen Diskursen zurückgedrängt wurden, in der fiktionalen Literatur aber bezeichnenderweise fortbestehen; besonders ausgeprägt ist die Vorliebe für alchemistische Zusammenhänge in der deutschsprachigen Literatur um 1800. In einer überzeugenden, vielschichtigen Interpretation von Goethes Faust und E. T. A. Hoffmanns Sandmann zeigt er die Alchemie unter Berücksichtigung der Relation von Text und Bild in ihrer neuen poetologischen, medienreflexiven Dimension.

Dirk Uffelmann analysiert in seinem Beitrag die Verflechtung von Wissen und Nicht-Wissen in Sienkiewicz' Knabenroman $W$ pustyni $i$ w puszczy und zeigt, wie die verschiedenen Facetten des (Nicht-)Wissens sich auf einander beziehen, einander ergänzen oder als koloniales Wissen und Opferwissen einander in Konflikt geraten.

Ein Spezifikum der ,russischen Philosophie‘ ist, wie Hanuš Nykl schreibt, eine vorherrschende Absenz von systematischen Fachdiskursen. Die Vermittlung des am Publikum orientierten russischen Denkens und deren Popularisierung erfolgt mit Blick auf die Umsetzung der Ideen in der realen Welt vielmehr durch Literatur. Der Fokus liegt auf der Wechselbeziehung von Philosophie und literarischem Schaffen in den Werken von Aleksandr S. Chomjakov, Konstantin N. Leont'ev und Vladimir S. Solov'ev.

Das Thema von Jan Vorels weitgreifender Studie ist die ,ästhetisch-philosophische Metamorphose des tschechischen und russischen Modernismus“, der sich vom „Kantschen Modell der autonomen Schönheit zur Hegelschen Darstellung der absoluten Idee in der Kunst" wandte. Systematisch und anschaulich werden die verschiedenen Entwicklungsphasen des Symbolismus dargestellt, die von einer L'art pour l'art-Ästhetik zum theurgischen Konzept führen.

Isabell Wünsche befasst sich mit Nikolaj Kul'bins und Wassily Kandinskys kunsttheoretischen Überlegungen zum künstlerischen Schaffensprozess, deren Genese und Inhalte die Autorin in ihrer subtilen, klar aufgebauten Untersuchung in einen breiteren wissenschaftstheoretischen Kontext stellt. Die Arbeiten beider Künstler sind geprägt durch einen subjektiv-intuitiven und synästhetischen Zugang zur Kunst.

Miroslav Olšovský reflektiert in seinem bisweilen ,fragmentarisch' anmutenden Essay über verschiedene Aspekte der Philosophie des im Westen kaum bekannten russischen Philosophen und Musikologen Jakov Ruskin und setzt dessen Denken in Bezug zu europäischen Philosophen wie Husserl, Heidegger und Sartre. Eine wichtige Erkenntnis, die uns der Autor in Auseinandersetzung mit dem ,anderen' russischen Denken vermittelt, ist die Einschränkung kreativen Denkens durch „Unterdrückung und Gewalt“, die sich in einer vorweg bestimmten intentionalen Fixierung ,auf einen Gegenstand“ - oder, um ein aktuelles Beispiel zu nennen, im repressiven Systemzwang des Wissenschaftsbetriebs vieler Länder - manifestiert. 
Das im Jahre 1953 veröffentlichte Essay Der gordische Knoten des kontrovers rezipierten Autors Ernst Jünger ist Gegenstand von Siegfried Ulbrechts bemerkenswertem Aufsatz, in dem er Jüngers mythisierendes Verständnis von ,West' und ,Ost' als ,Freiheit“ und ,Macht' in den verschiedensten Dimensionen darlegt. Jüngers dichotomisches Geschichtsdenken wird nicht nur mit den Ideen von Bachofen, Niekisch und Wittvogel in Bezug gesetzt, sondern bietet auch ein Erklärungsmodell für aktuelle politische Verhältnisse.

Ausgehend von einer profunden Auseinandersetzung mit der einschlägigen Forschungsliteratur untersucht Helena Ulbrechtová die bislang kaum beachteten Parallelen zwischen zwei Romanen von Susan Taubes und Marija Rybakova. Korrespondenzen konstatiert sie u. a. sowohl auf narrativer Ebene als auch im philosophischen Subtext, der in beiden Werken eine jeweils spezifische Ausprägung des gnostischen Denkens offenlegt. Dabei erschöpft sich die Analyse des philosophischen Kontextes nicht allein im Gnostizismus, sondern verweist weiterführend auf Reflexe von Freuds Psychoanalyse und C. G. Jungs Archetypen.

Die Mehrstimmigkeit des Wissens in Thomas Stangls historischem Afrika-Roman Der einzige Ort wird auf kreative Weise in einem Dialog zwischen Karin S. Wozonig, einer ,professionellen', mit literaturwissenschaftlichem Wissen ausgestatteten Leserin, und dem Autor auf eine Metaebene transponiert. Durch die Konfrontation von Leser- und Autorhorizont generiert die Lektüre des Werkes eine, Wissensvielfalt' der Textdeutung.

Abgerundet wird das Buch durch ein Verzeichnis der mitwirkenden AutorInnen und ein Personenregister. Um der besseren Übersicht willen wäre ein Literaturverzeichnis am Ende der einzelnen Beiträge wünschenswert, auch hätten einige Aufsätze etwas gestrafft werden können, doch leistet die vorliegende Monographie alles in allem einen wertvollen innovativen wissenschaftlichen Beitrag, der aus transdisziplinärer Perspektive unser Wissen über, Wissen und, in oder durch Literatur' in bedeutendem Maße erweitert und zu neuen Forschungen anregt. 